\title{
Association between dietary vitamin $D$ and calcium intakes and bone health outcomes in UK African-Caribbean women
}

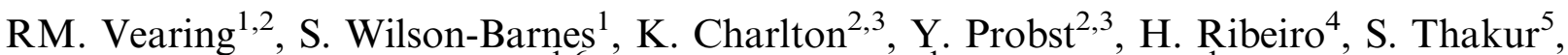 \\ M. Mendes ${ }^{1,6}$, SA. Lanham-New ${ }^{1}$ and KH. Hart ${ }^{1}$ \\ ${ }^{1}$ School of Biosciences and Medicine, Faculty of Health and Medical Sciences, University of Surrey, Guildford, \\ GU27XH, UK, \\ ${ }^{2}$ School of Medicine, Faculty of Science Medicine and Health, University of Wollongong, Wollongong, 2522, Australia, \\ ${ }^{3}$ Illawarra Health and Medical Research Institute, Wollongong, NSW 2522, Australia, \\ ${ }^{4}$ School of Public Health, University of São Paulo, São Paulo, 01246-904, \\ ${ }^{5}$ College of Veterinary Medicine, North Carolina State University, Raleigh, NC 27695, USA and \\ ${ }^{6}$ School of Nutrition, Faculty of Health Sciences, University of Brasilia, Brasilia, 70910-900, Brazil
}

African Caribbean (AC) populations are 'at risk' of vitamin D deficiency in the UK ${ }^{(1)}$. Vitamin D dietary intake is especially important in winter months, when cutaneous production of vitamin D from UVB rays is not possible in Northern latitudes. (1). Vitamin D together with calcium play a crucial role in bone health ${ }^{(1)}$. The aim of this study was to assess the dietary intake of vitamin $\mathrm{D}$ and calcium of the AC population and the association with bone health outcomes.

A cross-sectional study was carried out at the University of Surrey, UK, in winter 2020. Healthy, self-identified AC women aged $>18$ years, not in menopause or taking vitamin D containing supplements, were recruited. Dietary intakes were assessed using 4-day estimated food diaries, analysed via Nutritics software (v5.099), whilst Dual Energy X-ray Absorptiometry scans of the whole body and left hip were used to assess bone health and analysed using Hologic QDR (Hologic Inc., S/N 200071, USA). Anthropometric measurements and blood samples were taken and UVB exposure was assessed. The relationships between vitamin D and calcium intakes and bone heath (bone mineral density and bone mineral composition) were analysed by Pearson correlation. Statistical analysis was performed using SPSS (IBM, v27).

The study was approved by NHS Health Research Authority; REC number: 19/LO/1773 and was registered with ClinicalTrials.gov ID: NCT04179370.

$\mathrm{n}=15$ young $\mathrm{AC}$ women have been recruited for this study, mean age 22.37 (SD 2.63). Preliminary analysis identified low vitamin $\mathrm{D}$ and calcium intakes $2.3 \mu \mathrm{g} / \mathrm{day}$ (SD 1.4), and $489.4 \mathrm{mg}$ /day (SD 209.3) respectively with only $23.0 \%$ of participants meeting the RNI for vitamin D $(10.0 \mu \mathrm{g} / \mathrm{d})$, and $70.0 \%$ meeting the RNI for calcium $(700.0 \mathrm{mg} / \mathrm{d}){ }^{(2)}$. All participants were classified as having normal bone densitometry, according to total hip and whole-body $\mathrm{t}$ and z-scores. No significant associations were found between vitamin $\mathrm{D}$ and calcium and bone health, except a significant moderate negative correlation between vitamin D and whole-body BMD ( $\mathrm{p}<0.05$, $\mathrm{r}=-.515)$.

Interim analysis suggests poor intakes of nutrients relevant to bone health in this population. In comparison to the National Diet and Nutrition Survey data, this population have poorer intake of both vitamin D and calcium (2.5 $\mu \mathrm{g} / \mathrm{day}$ (SD 1.8), and 746mg/day (SD 288) respectively ${ }^{(3)}$. Despite this, no detriment to bone health was seen, however it may be premature to see the effects of poor vitamin D and calcium intake, due to the young age of participants. Given this population is at high risk of vitamin D deficiency due to possessing a darker skin type and living at a high latitude with low sun exposure, it is important to follow up and carry out further analysis on UVB exposure and serum $25(\mathrm{OH}) \mathrm{D}^{(1)}$. The study will be repeated in 2021 to increase sample size.

\section{Acknowledgments}

This work is part of the $\mathrm{PhD}$ of $\mathrm{RV}$, which is funded by the Universities Global Partnership Network, co-supervised by the Universities of Surrey and Wollongong.

\section{References}

1. Scientific Advisory Committee on Nutrition (2016) SACN Vitamin D and health report (2016) [online] Available:https://assets.publishing.service. gov.uk/government/uploads/system/uploads/attachment_data/file/537616/SACN_Vitamin_D_and_Health_report.pdf.

2. Public Health England (2016) Government Dietary Recommendations [online] Available:https://assets.publishing.service.gov.uk/government/ uploads/system/uploads/attachment_data/file/618167/government_dietary_re commendations.pdf.

3. National Diet and Nutrition Survey (2018) [online] Results from Years $\overline{7}$ and 8 (combined) of the Rolling Programme (2014/2015 to 2015/2016) Available:https://assets.publishing.service.gov.uk/government/uploads/system/uploads/attachment_data/file/699241/NDNS_results_years_7_ and_8.pdf. 